\title{
Low-Jitter Trigger System for Pulse Calibration and Intercomparison of High-Speed Samplers
}

\author{
N. G. Paulter
}

\begin{abstract}
A low-jitter ( $<1$ ps) trigger system for pulsewaveform-based calibration and intercomparison of high-speed samplers is described. The system uses a commercially available pulse generator and pulse splitter/delay line.
\end{abstract}

Index Terms - High-speed, pulse calibration, sampling oscilloscope, transition duration, trigger jitter, waveform recorder.

\section{INTRODUCTION}

A low-jitter trigger system that is used for pulse waveform calibration and interlaboratory intercomparison of highspeed samplers is described. For pulse calibration, the temporal characteristics of the high-speed sampler must be known. This is usually obtained using high-speed pulse generators [1], [2]. Furthermore, because these high-speed samplers are usually operated in an equivalent-time mode, the pulse generator (PG) and sampler must be accurately synchronized to each other. In addition, the sampler circuit requires that the PG pulse arrive at the sampler after it has been triggered to start the sampling process. This delay can be accomplished by either: 1) placing a delay line between the pulse generator and sampler and triggering the pulse generator and sampler simultaneously or 2) delaying the trigger of the pulse generator relative to the oscilloscope and using no delay line. The problem of 1) relative to 2 ) is that the delay line causes pulse distortion and, therefore, affects the measured waveform. The advantage of 1) over 2) is that the pulse generator usually provides an output trigger and additional electronics are not required. For accurate characterization of high-speed samplers, however, minimizing pulse distortion is very important. In addition to providing the correct trigger delay, jitter (phase noise) between the PG trigger and sampler trigger must be considered because its effects on the observed pulse may be large. Regardless of its magnitude, however, trigger jitter does contribute to the observed transition duration (td) or bandwidth of the signal. (Recall, the td and equivalent bandwidth for a pulse are inversely proportional.) The system described here provides very low jitter outputs for synchronously triggering a high-speed pulse generator and the high-speed sampler of an oscilloscope or waveform recorder.

The best way to see how trigger jitter affects the observed td is to consider the following equation (an approximation based on Gaussian functions) in which the transition durations of the

Manuscript received January 8, 1997; revised December 10, 1998. The author is with the National Institute of Standards and Technology, Gaithersburg, MD 20899 USA.

Publisher Item Identifier S 0018-9456(98)09894-5. contributing elements are added in quadrature [3]

$$
t_{\mathrm{obs}}=\sqrt{t_{\mathrm{gen}}^{2}+t_{\mathrm{osc}}^{2}+t_{\mathrm{jit}}^{2}}
$$

where:

$t_{\text {olss }}$ observed td;

$t_{\text {gen }} \quad$ td of the pulse generator output pulse;

$t_{\mathrm{osc}}$ oscilloscope $\mathrm{td}$;

$t_{\text {jit }} \quad$ equivalent $\mathrm{td}$ of the jitter.

All of these time durations are for step-like pulses or responses. These time durations must be measured with a consistent reference on the pulse, such as the 10-90\% td (which is the time it takes for the pulse or step response to go from its $10 \%$ amplitude level to its $90 \%$ amplitude level), the $20-80 \%$ td, or some other convenient interval. If impulse-like pulses are used to characterize the sampler temporal characteristics instead of step-like pulses, then the width of the impulse-like waveforms must be converted to an equivalent step-pulse td. For example, for a Gaussian impulse and its integral [also known as the error function (erf)], the td of the erf $\approx 0.92$ full width at half maximum (FWHM) of the impulse. (The FWHM is the width of the impulse at half its peak amplitude.) The $t_{\mathrm{jit}}$ is called an equivalent td because it is the td of a filter circuit that would yield the same $t_{\text {obs }}$ as the jitter. For jitter with a Gaussian amplitude distribution $t_{\mathrm{jit}}(10-90 \%)$ is related to the jitter standard deviation $\sigma_{\text {jit }}$ by

$$
t_{\mathrm{jit}} \approx 2.56 \sigma_{\mathrm{jit}}
$$

where 2.56 is the difference between the arguments for the erf for erf $\approx 0.1$ and erf $\approx 0.9$ [4].

\section{TRIGgER SySteM DESCRIPTION AND MEASUREMENT RESULTS}

We can use (1) and (2) to approximate the level of jitter acceptable for a particular application. For example, if we want $t_{\mathrm{jit}}$ to contribute no more than $1 \%$ to $t_{\mathrm{obs}}$ then, ignoring $t_{\mathrm{gen}}$, $t_{\mathrm{jit}} \leq 0.14 t_{\mathrm{osc}}$, or $\sigma_{\mathrm{jit}} \leq 0.055 t_{\mathrm{osc}}$. So, for a $20-\mathrm{GHz}(17.5-\mathrm{ps}$ td) oscilloscope $\sigma_{\text {jit }} \leq 0.96$ ps and for a $50-\mathrm{GHz}$ oscilloscope (7-ps td) $\sigma_{\mathrm{jit}} \leq 0.39 \mathrm{ps}$. The reason $t_{\mathrm{jit}} \leq 0.01 t_{\mathrm{obs}}$ is used for this example is that errors in the approximation of the td of high-speed oscilloscopes of $1 \%$ or less are usually acceptable. If system jitter is greater than $0.14 t_{\mathrm{osc}}$ and its functional form known, the jitter may be removed by waveform reconstruction [5]-[7]. The transition duration and other waveform parameters would then be extracted from the reconstructed record. However, the reconstruction process may be unstable and produce unlikely results in the reconstructed waveform, such 


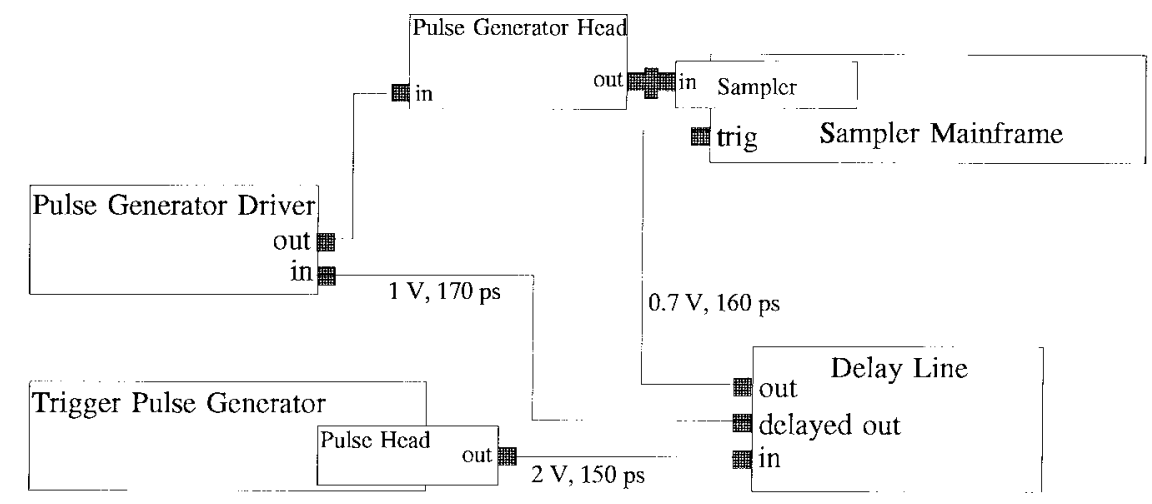

Fig. 1. Diagram of measurement system including nominal amplitudes and transition durations of trigger signals. The solid heavy line indicates the multiline connection between the pulse generator driver and the remote pulse generator head and the solid light lines indicate the trigger signal paths.

as large and rapid oscillations. Furthermore, the reconstruction process introduces additional uncertainties into the reconstructed record. A trigger system is described here that has low enough jitter to allow the jitter effects to be ignored entirely.

Jitter measurements were made using three different highspeed equivalent-time sampling oscilloscopes, a high-speed electrical pulse generator, and the trigger system (described below). Two of the samplers $S_{A}$ and $S_{B}$ have 2-GHz bandwidths (17.5-ps td) and were made by different manufacturers (Manufacturers A and B), and the third sampler $S_{C}$ (from Manufacturer A) has a 50-GHz bandwidth (7-ps td). The pulse generator produced step-like pulses with transition durations of nominally $15 \mathrm{ps}$.

The trigger system consists of the trigger pulse generator unit, a Berkeley Nucleonics Corporation Model 201E pulse generator module with the Model 6040 pulse generator mainframe, and the delay unit, a Picosecond Pulse Laboratories (PSPL) Model 5056 delay line (see Fig. 1). All components are connected using as-short-as-possible lengths of semirigid coaxial cable $(2.18-\mathrm{mm}$ diameter) and SMAtype connectors. The pulse generator head (Tektronix Model 067-1338) is connected directly to the sampler using a male-to-male connector. The amplitude levels and transition durations indicated in Fig. 1 are nominal values. The trigger pulse generator provides step-like pulses with 150-ps transition durations that are adjusted to have a nominal amplitude of $2 \mathrm{~V}$. These 150-ps pulses are delivered to the trigger delay unit which has a delay between its two output ports (one for the delayed pulse and one for the undelayed pulse) of approximately $56 \mathrm{~ns}$. The circuit path corresponding to the undelayed output has a 45-ps td and impresses a 9-dB attenuation on the input signal to yield a $0.7-\mathrm{V} 160-\mathrm{ps}$ pulse at the undelayed output port. The circuit corresponding to the delayed output has an 80ps td and impresses a 6-dB attenuation on the input signal to yield a 1-V 170-ps pulse at the delayed output port. If desired, the delayed output can be reduced to the same level as the undelayed output by using attenuators. Larger trigger signals can be obtained by adjusting the trigger pulse generator output. The increase in td for both output ports of the delay unit is caused by delay line dispersion and the compensation circuitry in the PSPL Model 5056.
The observed $\sigma_{\mathrm{jit}}$ values for the measurement configuration shown in Fig. 1 for the three different samplers $S_{A}, S_{B}$, and $S_{C}$ are

$$
\begin{array}{ll}
S_{A}(17.5 \mathrm{ps}) & 0.515 \mathrm{ps} \pm 0.063 \mathrm{ps} \\
S_{B}(17.5 \mathrm{ps}) & 0.9 \mathrm{ps} \pm 0.0 \mathrm{ps} \text { and } \\
S_{C}(7.0 \mathrm{ps}) & 1.475 \mathrm{ps} \pm 0.011 \mathrm{ps} .
\end{array}
$$

The standard deviation for measurements using $S_{B}$ is zero because of the limited measurement resolution. We can see that the observed jitter is very low for the 17.5-ps samplers, especially for $S_{A}$ and that the jitter observed for $S_{A}$ and $S_{B}$ satisfy our requirement that $\sigma_{\mathrm{jit}} \leq 0.055 t_{\mathrm{osc}}$. For the 7-ps sampler $S_{C}$ the jitter appears excessively large. It can be shown, however, that observed measurement jitter is a combination of signal amplitude noise and trigger jitter [5]. Consequently, the actual trigger jitter is less than that observed and reported above for all three samplers. The reason $S_{C}$ may show significantly larger jitter effects than $S_{A}\left(S_{A}\right.$ and $S_{C}$ have a common architecture) is the increased signal bandwidth of $S_{C}$ relative to $S_{A}$ and the concomitant increase in $S_{C}$ 's noise characteristics. The rms amplitude noise for $S_{A}$ was measured to be about $3 \times 10^{-4} \mathrm{~V}$ and for $S_{C}$ it was about $1.9 \times 10^{-3} \mathrm{~V}$. Although only two bandwidths are examined here, the ratio of the observed jitter for $S_{A}$ and $S_{C}$ is approximately equal to their bandwidth ratio (which is 2.5 ).

In conclusion, a very low jitter trigger system suitable for high-speed pulse calibration and measurement was assembled and evaluated. This system utilizes commercially available electrical components ${ }^{1}$ and allows sampler-to-pulse-generator jitter of less than 1 ps for $20-\mathrm{GHz}$ samplers (from manufacturers A and B) and under 1.5-ps jitter for a 50-GHz sampler (from manufacturer $\mathrm{A}$ ).

\section{REFERENCES}

[1] W. L. Gans, "Dynamic calibration of waveform recorders and oscilloscopes using pulse standards," IEEE Trans. Instrum. Meas., vol. 39, pp. 952-957, Dec. 1990.

\footnotetext{
${ }^{1}$ Commercial instruments are identified by manufacturer's name and/or model number in this paper so that procedures and tests discussed herein can be described adequately. In no case does such identification imply recommendation or endorsement by the National Institute of Standards and Technology.
} 
[2] D. Henderson and A. G. Roddie, "Calibration of fast sampling oscilloscopes," Meas. Sci. Technol., vol. 1, pp. 673-679, 1990.

[3] H. W. Johnson and M. Graham, High-Speed Digital Design, A Handbook of Black Magic. Englewood Cliffs, NJ: PTR Prentice-Hall, 1993, pp. 399-407.

[4] M. Abramowitz and I. E. Stegun, Eds., Handbook of Mathematical Functions with Formulas, Graphs, and Mathematical Tables. Washington, DC: U.S. Government Printing Office, 1972.

[5] W. L. Gans, "The measurement and deconvolution of time jitter in equivalent-time waveform samplers," IEEE Trans. Instrum. Meas., vol. 32, p. 126, Mar. 1983

[6] N. G. Paulter, "A causal regularizing deconvolution filter for optimal waveform reconstruction," IEEE Trans. Instrum. Meas., vol. 43, p. 740, Oct. 1994.

[7] A. Bennia and S. M. Riad, "Filtering capabilities and convergence of the Van-Cittert deconvolution technique," IEEE Trans. Instrum. Meas., vol. 41, p. 246, Apr. 1992.

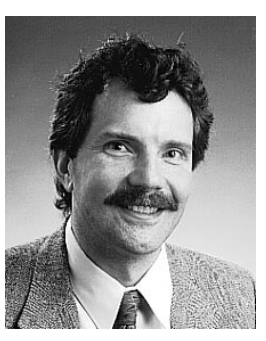

N. G. Paulter received the M.S. degree in chemistry from the University of New Mexico in 1988 and in electrical engineering from the University of Colorado in 1990.

He was with Los Alamos National Laboratory, Los Alamos, NM, from 1980 to 1989 and was involved in the study of fast electrical phenomena and in the development of high-speed photoconductors for use as ultrafast light detectors and sampling gates. In 1989 he joined the National Institute of Standards and Technology (NIST), Boulder, CO, to develop transient pulse measurement techniques and analysis. He is presently with NIST in Gaithersburg, MD. His present research interests include semiconductor physics, materials properties, electro-optics, ultrafast electronic phenomena, and waveform/data processing and analysis. 